Focus to an attribute with verbal or numerical quantifiers affects the attribute framing effect

Dawn Liu, Marie Juanchich, Miroslav Sirota, University of Essex

Note. This is an accepted manuscript for an article to be published in the journal Acta Psychologica; the current version might differ slightly from the published version.

\begin{abstract}
People find positive attribute frames (e.g., 75\% lean) more persuasive than negative ones (e.g., 25\% fat). In three pre-registered experiments, we tested whether this effect would be magnified by using verbal quantifiers instead of numerical ones (e.g., ‘high \% lean' vs. ‘75\% lean'). This moderating effect of quantifier format was predicted based on previous empirical work and two non-exclusive accounts of framing effects. First, verbal quantifiers are presumed to be a more intuitive format than numerical quantifiers, so might predispose people more to judgement biases such as the framing effect. Second, verbal quantifiers draw a greater focus to the attributes they describe. This could provide a linguistic signal that the positive frame is better than the negative one. In three experiments, we manipulated the attribute frame (positive or negative) and the quantifier format (verbal or numerical) betweensubjects, and quantity pairs (e.g., $5 \%$ fat and $95 \%$ lean or $25 \%$ fat and $75 \%$ lean) within-subjects. We also tested if participants focused more on the attributes in the frame, by measuring whether participants selected causal sentence completions about the beef that focused on why it had fat meat or lean meat. Results showed a robust framing effect, which was partially mediated by the focus of the sentence completions. However, the verbal format did not increase the magnitude of the framing effect. These results suggest that a focus on the attribute contributes to the framing effect, but contrary to past work, this focus is not different between verbal and numerical quantifiers.

Keywords: attribute framing; framing effect; quantifiers; associative encoding; pragmatic inference; focus
\end{abstract}




\section{Focus to an attribute with verbal or numerical quantifiers affects the attribute}

\section{framing effect}

The description, or 'frame', that people choose to present an item changes how others judge that item (Tversky \& Kahneman, 1981). Logically speaking, it should not matter whether one describes a beef as ' $25 \%$ fat' or ' $75 \%$ lean', as these are mathematically equivalent. However, people will judge a $75 \%$ lean beef as more desirable than a $25 \%$ fat one (Levin \& Gaeth, 1988). This 'attribute framing effect', where the positive or negative presentation of an item's attribute affects the evaluation of the item although these presentations are logically equivalent, has been robustly demonstrated across multiple domains, including performance evaluation (Kreiner \& Gamliel, 2017; Leong, McKenzie, Sher, \& Müller-Trede, 2017), health decisions (Krishnamurthy, Carter, \& Blair, 2001), enjoyment of events, (Isaac \& Poor, 2016), and even mate choice (Saad \& Gill, 2014).

Although framing effects - attribute or otherwise 1 - have been widely studied for decades, the question about what moderates the effect (i.e., factors that increase or decrease its size), and why, remains relevant because it provides insight into the cognitive processes and contexts driving the behaviour (Gal \& Rucker, 2018; Maule \& Villejoubert, 2007). One potential moderator that has received little empirical follow-up is the format of a quantifier: whether the amount of the attribute is in numerical (e.g., ‘75\% lean') or verbal (e.g., ‘high \% lean') format. Previous work suggests that using a verbal quantifier could magnify the framing effect size compared to numerical quantifiers (Welkenhuysen, Evers-Kiebooms, \& d'Ydewalle, 2001), however this work has yet to be replicated on a larger scale, and the reasons

1 Attribute framing is one of a larger class of framing effects, involving risky choice framing and goal framing (for a review of framing typology, see Levin, Schneider, \& Gaeth, 1998). In this paper, we refer specifically to the framing effect in attribute framing only. 
for a moderated effect are yet to be explained. The goal of this paper was to retest how quantifier format might moderate the framing effect, and explain this moderating role using two competing, but non-exclusive, accounts of the framing effect.

\section{The role of quantifier format in the framing effect}

A framing scenario is typically constructed using numerical quantifiers, with which it is easy to create mathematical complements. However, some studies showed that the framing effect also occurred with verbal quantifiers (Welkenhuysen et al., 2001; see also Reyna \& Brainerd, 1991, for an example in risky choice framing). One past study showed that verbal quantifiers produced a greater framing effect than their numerical equivalents (Welkenhuysen et al., 2001). In this case, Welkenhuysen et al. (2001)'s participants decided in a hypothetical scenario whether they would be willing to take a prenatal diagnostic test for cystic fibrosis based on a positively or negatively framed chance presented in either numerical or verbal format - where the verbal format was constructed using the most common verbal translations of a $25 \%$ chance ('moderate') of a baby with the disease (negative frame) and $75 \%$ chance ('high') of a baby without the disease (positive frame). Participants were more willing to take the test in the negative frame-showing they judged this event more negatively than in the positive frame-but only in the verbal condition.

While this empirical evidence suggests that verbal quantifiers can magnify the framing effect, there is a methodological challenge in studying verbal framing, which the previous work has not addressed. A framing study relies on ensuring that the pairs of opposite frames are equal. However, while numerical frames are clearly mathematical complements, verbal frames are less clearly so. One might think that adjective pairs (e.g., 'low' and 'high') should form equivalent complements. However, these terms are not translated in a numerically complementary fashion 
(Berry, 2006; Budescu, Por, Broomell, \& Smithson, 2014; Liu, Juanchich, Sirota, \& Orbell, 2019). Further, individuals vary in their interpretation of verbal quantifiers (e.g., Berry, Knapp, \& Raynor, 2002; Budescu, Por, \& Broomell, 2012), creating a greater challenge for ensuring equivalence when comparing verbal frames to standard numerical ones. In this paper, we report three experiments that sought to tackle this challenge while testing the moderating role of quantifier format in a traditional attribute framing context.

\section{Why would verbal quantifiers magnify the framing effect?}

There are two reasons why one would expect a verbal quantifier to magnify the framing effect. First, verbal quantifiers are believed to trigger more intuitive processing compared to numerical ones (Liu, Juanchich, Sirota, \& Orbell, 2020; Windschitl \& Wells, 1996). Because the attribute framing effect is proposed to be an intuitive response to the positive affect automatically created for the 'lean' frame compared to the negative 'fat' frame (Levin, 1987), increasing intuitive processing should magnify this affect-based responding (Thomas \& Miller, 2012; Keysar, Hayakawa, \& An, 2012). If verbal quantifiers are more intuitive than numerical ones, they should therefore generate a larger framing effect than numerical quantifiers.

Second, verbal quantifiers possess an inherent quality additional to the amount they express: verbal quantifiers also increase a reader's focus to the attribute it describes (Sanford \& Moxey, 2003). Readers, for instance, detected changes in attributes more between statements (e.g., the change from most of the people to most of the population was detected more than the change from $80 \%$ of the people to $80 \%$ of the population) with verbal than numerical quantifiers (Moxey, 2017). This suggests that a statement such as 'beef with a high \% of lean meat' could increase people's attention on the lean meat available, compared to beef with ' $75 \%$ of lean 
meat'. This would result in a more positive judgement of the high $\%$ lean beef than the $75 \%$ lean (and vice versa for fat), thus producing a magnified framing effect for verbal quantifiers. In addition, verbal quantifiers can be less ambiguous than numerical quantifiers (Teigen \& Brun, 1995, 2000). For example, beef with 'a moderate $\%$ of fat meat' could evoke reasons for why meat is fat, compared to beef with ' $25 \%$ of fat meat' (which could suggest some reasons for the inclusion of lean meat; Teigen \& Brun, 2000). We therefore expected that verbal quantifiers could magnify the effect of positive or negative attributes compared to numerical quantifiers.

\section{Present research}

The three experiments reported herein were designed to systematically address whether the framing effect's magnitude increases when a verbal, rather than numerical, quantifier is used. We also tested whether this might be explained by verbal quantifiers increasing attention to the attribute in the frame (Sanford \& Moxey, 2003) — we investigated whether participants would select causal sentence completions that explained, for example, 'a low \% of fat meat' with a reasons focused on explaining the fat or lean meat content. In our studies, we used verbal quantifier constructions that should focus attention on the attribute (e.g., 'a low', as opposed to 'low'). Based on previous research comparing the focus to an attribute between verbal and numerical probabilities, we hypothesised that people would focus more on the attribute in the frame with verbal quantifiers than numerical quantifiers (Moxey, 2017; Teigen \& Brun, 1995, 2000). In line with the most recent scientific guidelines, all our methods, hypotheses, and analyses were pre-registered prior to conducting the experiments. The pre-registrations, materials, and data for the experiments are 
available on the Open Science Framework (OSF;

https://osf.io/bm6ad/?view_only=8dd90314fd4b4d3f977958177b6963bd).

\section{Experiment 1}

\section{Method}

Participants. The experiment was powered to detect an interaction with an expected effect size of $f=.10(\alpha=.05,1-\beta=.80$, minimum required sample size was 280 participants). Participants were sourced from a survey panel company $(N=363$; offered online vouchers for participation) and from a university undergraduate pool $(N=181$; rewarded with course credit). After excluding unfinished and careless responses according to a priori defined criteria (either finishing in less than one-third of the median completion time or failing to disagree with the attention check question, 'I have never brushed my teeth.'), the sample had 335 participants (194 from the survey panel, 161 undergraduates)2. Participants were 59\% female, $80 \%$ White, with an age range of $18-76$ years $(M=37.76, S D=17.30)$.

Design. The experiment used a 2 (frame: positive [lean] vs. negative [fat]) $\times 4$ (quantity pair, see Table 1 for the four levels) $\times 2$ (format: verbal vs. numerical) mixed design, with frame and format manipulated between-subjects and quantity pair within-subjects. Order of presentation was randomised.

Materials. We used the attribute framing context of fat vs. lean meat (Levin, 1987; Levin \& Gaeth, 1988), which has been replicated in many independent studies (Donovan \& Jalleh, 1999; Kim, Kim, \& Marshall, 2014; Kreiner \& Gamliel, 2017; Seta, McCormick, Gallagher, McElroy, \& Seta, 2010). By using a known manipulation, we were more confident of producing the main effect that we could then test for moderation.

2 The framing effect was not significantly different between samples, thus all results were analysed with both samples combined. 
Because the framing effect has been extended to different pairs of quantities (e.g., $25 \%$ vs. $75 \%, 20 \%$ vs. $80 \%$ ), with inconsistent findings as to whether using different mathematical complements cause a larger or smaller effect (studies reporting differences in effect size across different quantities: Janiszewski, Silk, \& Cooke, 2003; Kim et al., 2014; Sanford, Fay, Stewart, \& Moxey, 2002; studies reporting no differences: Jin, Zhang, \& Chen, 2017; Olsen, 2015), we tested a range of quantity pairs to ascertain if the predicted larger verbal framing effect would be robust across quantities.

Procedure. After providing informed consent, participants performed a translation task, where they provided verbal equivalents of the four numerical quantities used in the experimental frames. The purpose of this task was to create equivalent verbal and numerical frames for comparison across conditions. Participants selected for four numerical percentages the most appropriate verbal quantifier from a randomised drop-down list of 13 (see the Appendix for the full list). For example, they were told 'the beef contains $25 \%$ of fat meat', and they picked a word to complete the sentence: 'There is $a(n)$ percentage offat meat in the beef.' Participants also provided two filler translations of other food quantities (e.g. 'low \% calories') that served as distractions. Table 1 shows the most common translation of numerical quantities into verbal ones. Subsequently, in the verbal condition of the framing task, participants saw the verbal quantifier they had themselves selected during the translation task. 
Table 1.

Most common translations of numerical quantities into verbal quantifiers.

\begin{tabular}{|c|c|c|c|c|}
\hline & \multicolumn{2}{|c|}{ Numerical Frames } & \multicolumn{2}{|c|}{$\begin{array}{l}\text { Most common verbal translation } \\
\text { (with \% who selected it across experiments) }\end{array}$} \\
\hline & Positive & Negative & Positive frame & Negative frame \\
\hline Pair 1 & $95 \%$ lean & $5 \%$ fat & $\begin{array}{l}\text { A very high } \% \text { of lean } \\
\text { meat }(63-84 \%)\end{array}$ & $\begin{array}{l}\text { A low } \% \text { of fat meat } \\
(51-58 \%)\end{array}$ \\
\hline Pair 2 & $75 \%$ lean & $25 \%$ fat & $\begin{array}{l}\text { A high } \% \text { of lean meat } \\
\qquad(40-53 \%)\end{array}$ & $\begin{array}{l}\text { A medium } \% \text { of fat meat } \\
\qquad(37-48 \%)\end{array}$ \\
\hline Pair 3 & $50 \%$ lean & $50 \%$ fat & $\begin{array}{l}\text { A medium } \% \text { of lean } \\
\text { meat }(76-77 \%)\end{array}$ & $\begin{array}{l}\text { A very high } \% \text { of fat } \\
\text { meat }(41-49 \%)\end{array}$ \\
\hline Pair 4 & $25 \%$ lean & $75 \%$ fat & $\begin{array}{l}\text { A low } \% \text { of lean meat } \\
\qquad(62-67 \%)\end{array}$ & $\begin{array}{l}\text { A very high } \% \text { of fat } \\
\text { meat }(68-77 \%)\end{array}$ \\
\hline
\end{tabular}

After performing the translation task, participants completed a distraction task that required them to complete a sentence describing computer battery life or jeans shrinkage similar to the ones used in Teigen, Juanchich, \& Filkukova (2014).

After the distraction task, participants performed the following framing task for four different quantity pairs that were either verbal or numerical, and which were presented in randomised order to each participant. In the verbal condition, the quantifier would be the participants' own translation of the corresponding numerical one. Participants judged the healthiness of meat in the following vignette:

'You are given the following information about a 250g beef fillet:

The beef contains a [quantity] \% of [attribute] meat.' 
Participants made healthiness judgements on a Likert scale (1: very unhealthy, 7: very healthy). They also indicated how much they would be willing to pay for the meat (in pounds sterling). We excluded from analysis unrealistic willingness-to-pay amounts that were more than five standard deviations above the mean (i.e., over $£ 15$ ). Participants also completed this task for two filler items (cereal bars with different energy values).

Finally, participants reported their attitudes towards healthy eating (Steptoe, Pollard, \& Wardle, 1995), how frequently they used nutrition labels, and sociodemographic information, including weight and height. We obtained this data as a check that our sample did not have extreme views towards health and nutrition, which might affect their judgements of food healthiness. Participants had on average a healthy BMI $(M=24.99, S D=5.78)$ and slightly positive attitudes towards healthy eating $(M=4.89$ on a 7-point scale from negative to positive, $S D=.98)$. Fifty-three percent reported frequent use of nutrition labels. Our sample was therefore not unusual in these measures (Naughton, McCarthy, \& McCarthy, 2015).

\section{Results}

Traditional framing effect. We observed the framing effect for each quantity pair: participants rated the $\%$ lean meat as healthier and were willing to pay more for it than for the $\%$ fat meat. Figure 1 illustrates the mean distributions of healthiness judgements and willingness-to-pay values for each format in the four quantity pairs. Table 2 shows the mean difference in healthiness judgements (positive - negative) for the different quantity pairs: scores further from zero indicate stronger framing effects. As healthiness and willingness-to-pay were significantly correlated, we conducted a mixed MANOVA on healthiness judgements and willingness-to-pay, using frame and format as between-subjects factors and quantity pair as a within-subjects factor. The 
framing effect was only significant for healthiness judgements, and not for

willingness-to-pay, $F(1,331)=99.40, p<.001, \eta 2 \mathrm{P}=.23, F(1,331)=.06, p=.815$, $\eta_{2 P}<.001$, respectively.

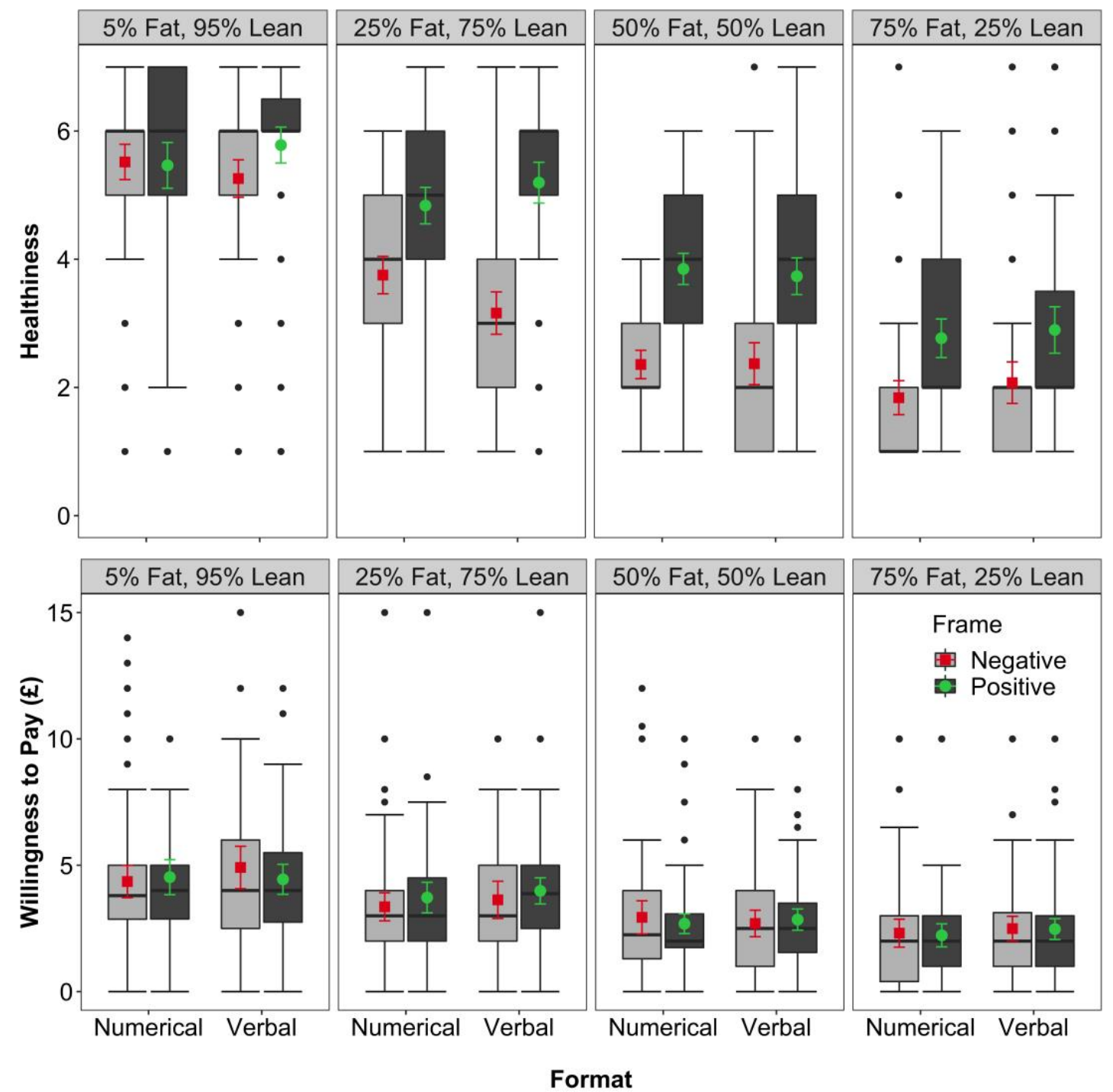

Figure 1. Means and distributions of healthiness judgements and willingness-to-pay values across verbal and numerical formats for four quantity pairs in Experiment 1.

Note. The point plots (red and green) give the means and $95 \%$ confidence intervals of participants' responses, and the box-and-whisker plots show the overall distributions of the responses. Framing effects (difference between positive and negative frame) were only significant for healthiness judgements. 
Table 2.

Magnitude of framing effect (positive frame minus negative frame) for healthiness judgements across quantity combinations in Experiments 1-3.

\begin{tabular}{|c|c|c|c|c|c|}
\hline \multirow{4}{*}{$\begin{array}{l}\text { Quantity } \\
\text { pair }\end{array}$} & \multicolumn{5}{|c|}{ Framing effect magnitude } \\
\hline & \multicolumn{5}{|c|}{ (Mean judgement difference between lean and fat frame) } \\
\hline & Verbal & \multicolumn{2}{|c|}{ Numerical } & \multicolumn{2}{|c|}{ Overall } \\
\hline & $95 \% C I$ & $M$ & $95 \% C I$ & $M$ & $95 \%$ CI \\
\hline
\end{tabular}

Experiment 1 (7-point scale; $N=355)$

$5 \%$ fat $/ 95 \% \quad 0.53 * \quad 0.10,0.96 \quad-0.01 \quad-0.44,0.42 \quad 0.26 \quad-0.05,0.56$

lean

$25 \%$ fat $/ 75 \% \quad 2.10^{* * *} \quad 1.67,2.53 \quad 1.11 * * * \quad 0.68,1.54 \quad 1.60 * * * \quad 1.30,1.91$

lean

$50 \%$ fat $/ 50 \% \quad 1.40^{* * *} \quad 1.02,1.78 \quad 1.49 * * * \quad 1.11,1.87 \quad 1.45^{* * *} \quad 1.18,1.72$

lean

$75 \%$ fat $/ 25 \% \quad 0.83 * * * \quad 0.39,1.28 \quad 0.89 * * * \quad 0.45,1.34 \quad 0.86^{* * *} \quad 0.55,1.18$

lean

Experiment 2 (100-point scale; $N=442)$

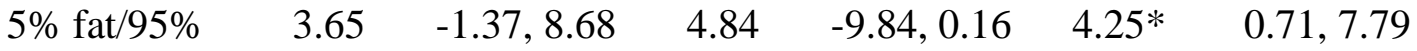

lean

$25 \%$ fat $/ 75 \% \quad 26.95 * * * 21.03,32.8734 .65 * * * 28.76,40.5430 .80 * * * \quad 26.62,34.98$

lean

Experiment 3 (11-point scale; $N=440$ )

$\begin{array}{lllllll}5 \% \text { fat } / 95 \% & 0.30 & -0.21,0.82 & 0.92 * * & 0.40,1.43 & 0.61 * * & 0.24,0.97\end{array}$

lean

$25 \%$ fat $/ 75 \% \quad 2.40 * * * \quad 1.84,2.96 \quad 3.04 * * * \quad 2.47,3.60 \quad 2.72 * * * \quad 2.32,3.12$

lean

Note. Larger scores indicate a larger framing effect (a greater difference in healthiness judgement between the lean and fat frame). Significant mean differences are indicated for $p<.05^{*}, p<.01^{* *}$, and $p<.001^{* * *}$. 


\section{Quantifier format moderated the framing effect for only one out of four}

quantity pairs. The format of the quantifiers affected framing differently across the quantity pairs (see Table 2$), F(3,975)=4.05, p=.007, \eta_{2} \mathrm{P}=.01$. The $75 \%$ lean vs. $25 \%$ fat pair produced a significantly larger framing effect in the verbal than the numerical format, $F(1,331)=9.49, p=.002, \eta_{2 \mathrm{P}}=.028$. However, there was no significant format and frame interaction for the other three quantity pairs of $5 \%$ fat, $50 \%$ fat and $75 \%$ fat, $F(1,331)=3.54, p=.061, \eta_{2 \mathrm{P}}=.01(5 \%$ fat $) ; F(1,331)=0.21, p$ $=.647, \eta_{2 \mathrm{P}}=.001(50 \%$ fat $) ; F(1,331)=0.11, p=.741, \eta_{2 \mathrm{P}}=<.001$ (75\% fat $)$.

Variations in framing effect across quantifiers. In addition to interacting with the quantifier format, quantity pair also affected the size of the framing effect, as illustrated in Figure 1. The largest framing effect was observed for the $25 \%$ fat pair, and the smallest with the 5\% fat pair (see Table 2). This was also quantified by significant two-way interactions between frame and quantity pair for healthiness and willingness-to-pay, $F(3,975)=22.91, p<.001, \eta 2 \mathrm{P}=.07$ and $F(3,975)=3.48, p=$ $.015, \eta 2 \mathrm{P}=.01$.

\section{Discussion}

Experiment 1 only found limited support for our hypothesis that quantifier format would moderate the framing effect. Only the $25 \%$ fat quantity pair had the expected larger verbal than numerical framing effect on healthiness judgements. We also found that the size of the framing effect was not consistent across the different quantity pairs. The largest framing effect was found with the $25 \%$ fat $/ 75 \%$ lean pair, and the smallest (no significant effect) with the 5\% fat/95\% lean pair-though this was primarily due to the lack of framing effect with the numerical quantifier in this condition. One potential explanation for the reduced framing effect with this quantity pair (smaller in size for low \%, and non-existent for 5\%) is that the healthiness 
judgements were too close to ceiling, as this would be the healthiest beef in the set. Another explanation for the smaller framing effect is that quantifiers may direct readers instead to the complement of the attribute (Teigen \& Brun, 2003). A reader could focus on the fat present in beef with $25 \%$ fat meat, or the absence of fat in beef with $5 \%$ fat meat - and therefore the presence of lean meat. The latter points in the same direction as its complementary lean frame, reducing the framing effect. The corresponding verbal frame to 5\% fat, 'a low \% of fat meat', may be more ambiguous in pointing to fat. In principle, 'a low' should direct readers to the attribute, as its sentence construction is similar to verbal quantifiers that put a focus on the referenced attribute (e.g., 'a few'; Moxey and Sanford, 1986). However, Sanford and Moxey (2003) showed that 'low fat' (without the \%) shifted focus to a complementary fatfree attribute. Furthermore, quantifiers that typically direct readers to attributes (e.g., 'a few') also led to a focus on the complement set if readers believe there to be a lower amount than expected (Moxey, 2006; Moxey \& Filik, 2010). We therefore do not know whether 'a low \%' directed readers to the attribute or its complement.

Because our test of the format and frame interaction was not significant for the $5 \%$ fat pair, we cannot conclude whether the verbal and numerical frames produced different focal directions on attribute or complement. To address these issues, we sought to replicate the moderated framing effect and compare them between $5 \%$ fat meat (the smallest effect) and 25\% fat meat (the largest effect) using a more sensitive scale, and investigate whether a focus on the attribute explained a moderated framing effect.

\section{Experiment 2}

The goal of Experiment 2 was to test the effect of quantifier format on attribute framing, and to explain the effect and its variation. Our expectations were 
that verbal quantifiers would magnify the framing effect and, in addition, that the moderated framing effect could be explained by how much participants focused on the attribute in the lean frame vs. the attribute in the fat frame. To simplify our analysis, we focused on the two quantity combinations that produced the greatest difference in effect size (5\% and $25 \%$ fat). This also allowed us to test if the focus would be different for verbal quantifiers and their numerical counterparts: for example, if $5 \%$ of fat and a low $\%$ of fat would both focus on the attribute 'fat', or its complement, 'lean'. In addition, we sought to rule out a methodological artefact for differences in framing effect size. We accounted for the possibility that the 7-point Likert scale in Experiment 1 might lack sensitivity and result in a ceiling effect for the 5\% fat quantity pair (Voutilainen, Pitkäaho, Kvist, \& Vehviläinen-Julkunen, 2016).

\section{Method}

Participants. The experiment was powered to capture the interaction effect obtained in Experiment $1(f=.10, \alpha=.05,1-\beta=.80$, minimum required sample size was 433). Four hundred and forty-two participants (72\% female; $90 \%$ White; age range $18-80$ years, $M=35.98, S D=10.98$ ) were offered $£ 0.60$ to complete the 5minute experiment on Prolific Academic3. We used the same exclusion criteria as in Experiment 1. Participants had on average an overweight BMI $(M=27.29, S D=$ 8.99) and positive attitudes towards healthy eating $(M=5.10, S D=0.84)$; seventythree percent reported frequent use of nutrition labels.

Design. The design was the same as Experiment 1 (format and frame manipulated between-subjects; quantity pair within-subjects), however we only used the $5 \%$ and $25 \%$ fat pairs.

3 This payment amounts to a $£ 7.20$ per hour wage, which is above the minimum wage recommendation for survey panel studies. 
Materials and procedure. After providing informed consent, participants performed the translation task for the numerical quantifiers as in Experiment 1 (see Table 1). They then rated the healthiness of meat described in the same Experiment 1 vignette for each quantity pair (shown in randomised order to each participant) on a sliding scale that increased from 0-100 in invisible increments of one. Seven descriptors were spaced over the scale (from very unhealthy to very healthy). After this, participants were presented again with the vignettes and given the following sentence completion task:

Pick the option that makes the most sense to complete the sentence:

The beef has [quantity] \% of [attribute] meat because ...

A the cow was grain-fed and developed a lot of fatty tissue [fat focus]

$B \quad$ the cow was grass-fed and developed a lot of lean muscle [lean focus]

The two options were presented in a random order. One option always focused on the presence of the attribute in the vignette (e.g., option A in the example for fat meat), while the other focused on its complement (e.g., option B for fat meat; vice versa for lean meat). At the end of the experiment, participants completed the same demographic survey as in Experiment 1.

\section{Results}

We ran a pre-registered moderated mediation analysis for each quantity pair to assess whether the effect of frame on healthiness judgement was mediated by a focus to the lean attribute (vs. the fat one; 1000 simulations using the $\mathrm{R}$ package 'mediation'; Tingley, Yamamoto, Hirose, Keele, \& Imai, 2014). This allowed us to estimate and test the average causal mediation effect and average direct effect as moderated by quantifier format for each of the two quantity pairs. The middle columns of Table 3 report the mediation analyses for each quantity pair. 
Effects of format and framing. The results indicated that framing had a direct effect on healthiness judgement for both the $5 \%$ fat and $25 \%$ fat pairs. The traditional framing effect is depicted in the top panel of Figure 2, and showed that participants judged the lean meat as healthier than the equivalent quantity presented in terms of fat content. The effect of framing was significant overall for both the $5 \%$ fat and the $25 \%$ fat pairs, $b=4.88, p=.044,95 \%$ CI $[0.139 .63]$ (5\% fat); $b=19.30, p<$ $.001,95 \%$ CI $[13.26,25.35]$ (25\% fat). However, the framing effect was not significantly different between verbal and numerical format, whether it was the direct effect of frame on healthiness or the mediated effect through the focus. Therefore, contrary to our expectations, quantifier format did not result in variations in the framing effect. Nor did verbal quantifiers appear to produce significantly more focus on the attribute than the numerical quantifiers, as shown in the moderated mediation by non-significant interaction effects between frame and format on the mediator (focus), $b=0.80, p=.370,95 \%$ CI $[-0.94,2.62]$ (5\% fat); $b=0.44, p=.361,95 \% \mathrm{CI}$ $[-0.49,1.39]$ (25\% fat).

Focus on 'lean' (vs. 'fat') mediated the framing effect. We expected participants to focus more on the attribute in the frame rather than its complement, as indicated by causal sentence completions that explained the frame in terms of the presented attribute. As illustrated in the top panel of Figure 3, we found that participants did select more sentence completions with a lean focus when the beef was described as 75 (or high) \% lean, but more sentence completions with a fat focus when the product was described as 25 (or moderate) $\%$ fat. However, in the other quantity pair, where the beef was described as 95 (or very high) \% lean or 5 (or low) $\%$ fat, most participants selected sentence completions with a lean focus regardless of the frame. The mediation analysis showed that a greater focus on the lean attribute 
mediated the effect of framing on healthiness judgement for the $25 \%$ fat pair, but not the $5 \%$ fat pair, $b=7.80, p<.001(25 \%$ fat $), 95 \%$ CI $[5.05,10.66] ; b=-0.60, p=$ $.406,95 \%$ CI $[-2.17,0.97]$ (5\% fat). 
Table 3.

Beta coefficients for direct, indirect, and total effects in the mediation models for frame, format, and focus in Experiments 2 and 3, including tests of moderated mediation by format

\begin{tabular}{|c|c|c|c|c|}
\hline \multirow[b]{2}{*}{$\begin{array}{l}\text { Framing } \\
\text { effects }\end{array}$} & \multicolumn{2}{|c|}{ Experiment 2} & \multicolumn{2}{|c|}{ Experiment 3} \\
\hline & $\begin{array}{l}5 \text { (low) \% fat } \\
\text { vs. } 95 \text { (very } \\
\text { high) \% lean }\end{array}$ & $\begin{array}{l}25 \text { (moderate) } \\
\% \text { fat vs. } 75 \\
\text { (high) \% lean }\end{array}$ & $\begin{array}{l}5 \text { (low) \% fat } \\
\text { vs. } 95 \text { (very } \\
\text { high) \% lean }\end{array}$ & $\begin{array}{l}25 \text { (moderate) } \\
\% \text { fat vs. } 75 \\
\text { (high) \% lean }\end{array}$ \\
\hline $\begin{array}{l}\text { Total framing } \\
\text { effect }\end{array}$ & $\begin{array}{l}4.22[0.66, \\
7.61]\end{array}$ & $\begin{array}{l}30.67[26.42, \\
34.78]\end{array}$ & $\begin{array}{l}0.60[-0.11, \\
0.30]\end{array}$ & $\begin{array}{l}2.71[2.32, \\
3.11]\end{array}$ \\
\hline $\begin{array}{l}\text { Effect on focus } \\
\text { on lean frame } \\
\text { (direct) }\end{array}$ & $\begin{array}{l}-0.82[-2.15 \\
0.33]\end{array}$ & $\begin{array}{l}2.09[1.49, \\
2.72]\end{array}$ & $\begin{array}{l}0.47[-0.81 \\
1.86]\end{array}$ & $\begin{array}{l}2.61[1.94, \\
3.35]\end{array}$ \\
\hline $\begin{array}{l}\text { Effect on } \\
\text { healthiness } \\
\text { (direct) }\end{array}$ & $\begin{array}{l}4.82[1.50, \\
8.02]\end{array}$ & $\begin{array}{l}22.87[18.43, \\
27.31]\end{array}$ & $\begin{array}{l}0.55[0.18, \\
0.92]\end{array}$ & $\begin{array}{l}1.63[1.23, \\
2.08]\end{array}$ \\
\hline $\begin{array}{l}\text { Indirect effect } \\
\text { (causal } \\
\text { mediation) }\end{array}$ & $\begin{array}{l}-0.60[-2.17 \\
0.97]\end{array}$ & $\begin{array}{l}7.80[5.05, \\
10.66]\end{array}$ & $\begin{array}{l}0.05[-0.05, \\
0.16]\end{array}$ & $\begin{array}{l}1.08[0.79, \\
1.39]\end{array}$ \\
\hline \multicolumn{5}{|c|}{ Tests of moderated mediation } \\
\hline Direct effect & $\begin{array}{l}-0.03[-6.55, \\
6.54]\end{array}$ & $\begin{array}{l}-7.27[-16.00, \\
1.84]\end{array}$ & $\begin{array}{l}-0.58[-1.31 \\
0.15]\end{array}$ & $\begin{array}{l}-0.59[-1.46 \\
0.15]\end{array}$ \\
\hline Indirect effect & $\begin{array}{l}-1.23[-4.26, \\
1.61]\end{array}$ & $\begin{array}{l}-0.49[-5.22, \\
4.00]\end{array}$ & $\begin{array}{l}-0.02[-0.20, \\
0.15]\end{array}$ & $\begin{array}{l}-0.05[-0.52 \\
0.41]\end{array}$ \\
\hline
\end{tabular}

Note. $95 \%$ confidence intervals (indicated in square brackets) were generated using 1,000 bootstrap samples. Focus on the lean frame was tested as a mediator of the frame-healthiness relationship. 

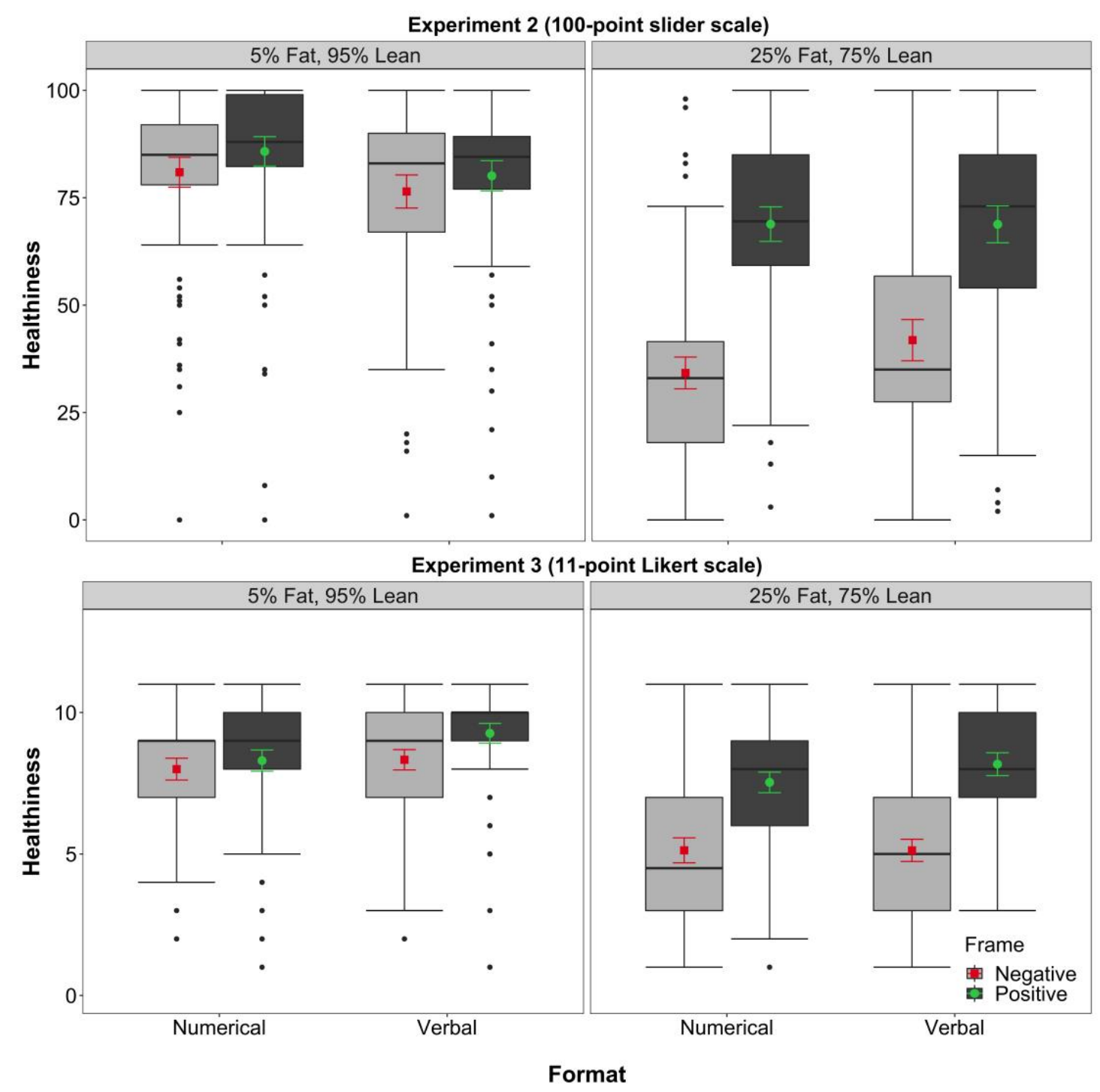

Figure 2. Means and distributions of healthiness judgements for verbal and numerical quantifiers in the 5\% and 25\% fat pairs in Experiments 2 (100-point scale) and 3 (11point scale), illustrating the framing effect between positive frames (green and dark grey) and negative frames (red and light grey).

Note. The point plots (red and green) give the means and 95\% confidence intervals of participants' responses, and the box-and-whisker plots show the overall distributions of the responses. Framing effects (difference between positive and negative frame) were significant for the $25 \%$ fat but not $5 \%$ fat combination. 
$5 \%$ fat $(95 \%$ lean) pair
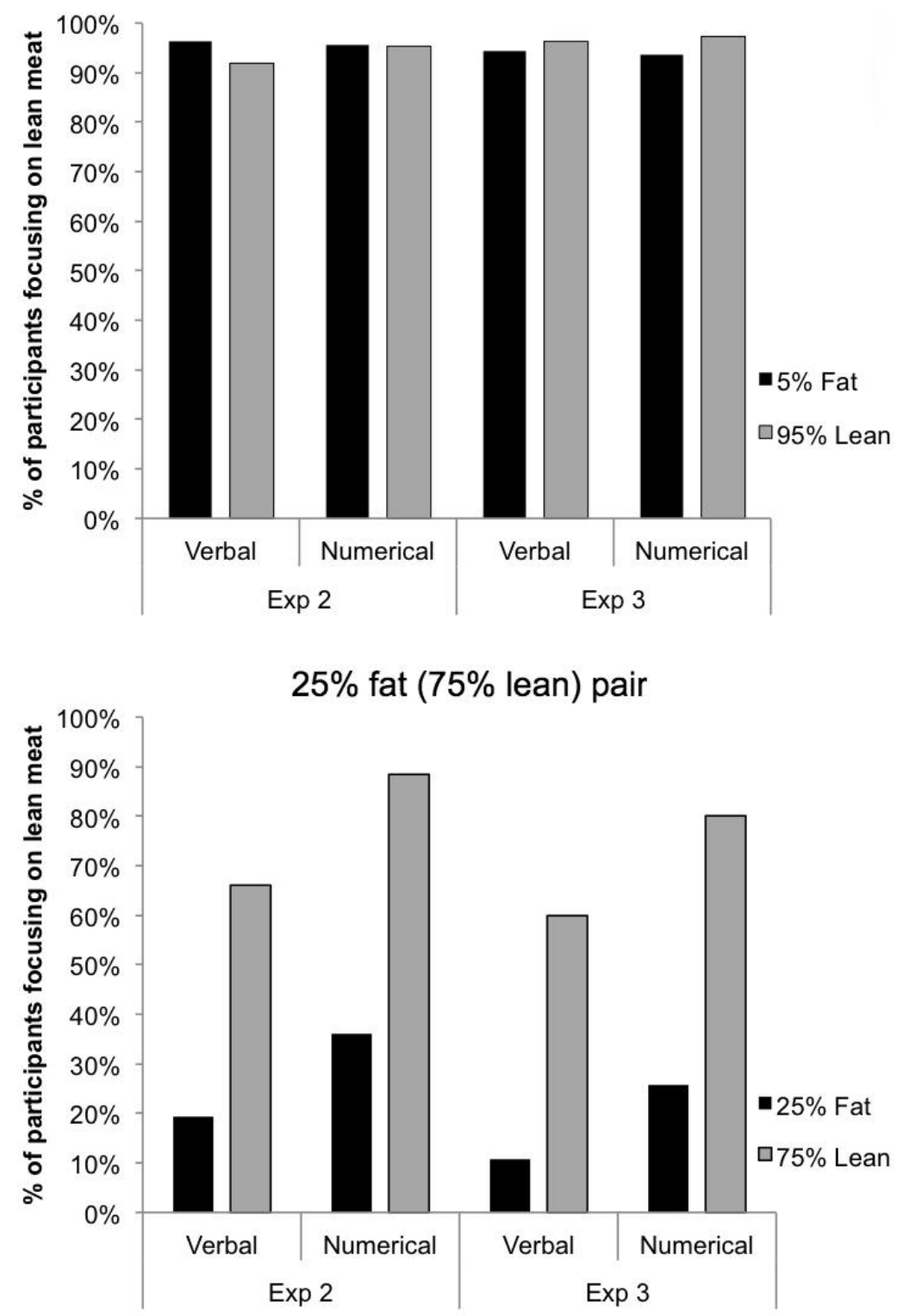

Figure 3. Percentage of participants who selected causal sentence completions with a reason focused on lean meat, grouped by format, frame, and quantity pair.

Note. The corresponding percentage (up to $100 \%$ ) is the percentage of participants who selected causal sentence completions with a reason focused on fat meat. Participants almost always focused on lean meat in the 5\% fat/95\% lean conditions, but the focus was more diverse in the $25 \%$ fat $/ 75 \%$ lean conditions. Differences in focus direction were not significant between quantifier formats. 


\section{Discussion}

Experiment 2 gave more evidence of the robustness of the framing effect, but we did not find evidence using a verbal quantifier magnified the framing effect for healthiness judgements. We expected that readers would focus on reasons justifying the attribute cited (e.g., 'lean' in a lean frame) more with the verbal quantifiers, and that this would explain a framing effect moderated by format. Our evidence only supported a mediating role of focus on the attribute for the $25 \%$ fat pairs, but it was not moderated by format. Both the numerical and verbal quantifiers had similar focus on the fat or lean attribute, respectively. In contrast, for the 5\% far pairs, participants focused on explaining how lean the product was, no matter whether it was described with a fat or lean frame. The numerical and verbal quantifiers also displayed similar focus on the lean attribute. 'A low $\%$ of fat' and ' $5 \%$ fat' thus seemed to function in a similar fashion to 'low fat', putting a focus on the complement (Sanford \& Moxey, 2003).

The fact that verbal quantifiers did not lead to larger framing effects in Experiment 2 could indicate that verbal quantifiers do not magnify framing effects compared to numerical ones. However, two factors in the experiment constrain this conclusion. First, we used a verbal to numerical translation task at the onset of the study that may have primed people to think about verbal quantifiers in a numerical way. This could have rendered verbal statements more similar to numerical ones. Second, the 100-point response scale, which aimed to avoid a ceiling effect to detect smaller judgement differences for the $5 \%$ fat pair, could have inadvertently caused an anchoring of judgements in the numerical condition to the corresponding scale points (e.g., $25 \%$ fat is $25 / 100$ healthy), thereby widening the response range between the numerical frames. In our next experiment, we sought to address these concerns. 


\section{Experiment 3}

Experiment 3 aimed to replicate Experiment 2 while overcoming two methodological limitations. First, we re-introduced a distractor task between the translation task and the actual framing evaluation task to reduce the likelihood that people were still thinking about their translations. Second, we adjusted the response scale to an 11-point Likert scale to reduce anchoring of responses to scale values. We tested again whether verbal quantifiers would magnify the framing effect, with focus on the lean attribute as a mediator. Based on the results from Experiment 1 and 2, we also predicted that the framing effect size would be larger for the $25 \%$ fat than the $5 \%$ fat pair.

\section{Method}

Participants. The experiment was powered to capture the previous interaction effects obtained $(f=.10, \alpha=.05,1-\beta=.80$, minimum required sample size was 433 participants). Four hundred and forty participants (66\% female; $89 \%$ White; age range 18-74 years, $M=33.90, S D=11.59)$ were offered $£ 1$ to complete the 10 -minute experiment on Prolific Academic. The exclusion criteria were identical to Experiments 1 and 2. Participants had on average a slightly overweight BMI $(M=$ 26.86, $S D=7.82)$ and positive attitudes towards healthy eating $(M=5.01, S D=0.94)$. Seventy-five percent reported frequent use of nutrition labels.

Design. The design was the same as Experiment 2, with format and frame manipulated between-subjects and quantity pairs manipulated within-subjects.

Materials and procedure. After providing informed consent, participants completed translations for the numerical quantifiers, including six filler translations (50\% fat, $75 \%$ fat, and four verbal-numerical translations for low $\%$ and high $\%$ 
risks). Participants then completed a distractor task where they described a graph about medical treatment outcomes. Subsequently, participants performed the healthiness judgement task for the two quantity magnitudes (5\% fat and $25 \%$ fat) in randomised order. Responses were made on an 11-point Likert scale (1: extremely unhealthy, 11: extremely healthy) so as to maintain the greater sensitivity of the rating scale while minimising the possibility of participants anchoring responses to the numerical quantifiers given. Following their healthiness judgements, participants performed the sentence completion task used in Experiment 2. Finally, they completed the demographic survey.

\section{Results}

We ran pre-registered mediation analyses for the effect of frame on healthiness judgement as mediated by focus on the lean attribute (vs. the fat one) and moderated by quantifier format. The right columns of Table 3 report the mediation analyses for each quantify pair in Experiment 3.

Effects of format and framing. Framing had a direct effect on healthiness judgement for both quantity pairs. The bottom panel of Figure 2 depicts the traditional framing effect, which was significant overall for both pairs, $b=0.60, p<.001,95 \%$ CI $[0.21,0.97]$ (5\% fat); $b=2.71, p<.001,95 \%$ CI $[2.32,3.11]$ (25\% fat). The tests of moderated mediation showed that quantifier format did not significantly affect the framing effect size. Quantifier format also did not affect the framing effect on the mediator, $b=0.42, p=.661,95 \%$ CI $[-1.47,2.40]$ (5\% fat); $b=-0.16, p=.736,95 \%$ CI $[-1.12,0.78]$. This provided additional evidence to Experiment 2 that contrary to expectations, the verbal quantifier did not magnify the framing effect or its mediation by a focus on the lean attribute. 
Focus on the lean frame mediated the framing effect. We replicated the effect of frame on sentence completions from Experiment 2. As illustrated in the bottom panel of Figure 3, participants selected more sentence completions with a lean focus for 75 (high) \% lean mean, but more sentence completions with a fat focus for 25 (moderate) \% fat meat. However, participants consistently selected sentence completions with a lean focus for both frames in the $5 \%$ fat pair. A greater focus on the lean attribute mediated the framing effect on healthiness judgement for the $25 \%$ fat pair, but not the $5 \%$ fat pair, $b=1.08, p<.001,95 \%$ CI $[0.79,1.39](25 \%$ fat $) ; b=$ $0.05, p=0.280,95 \%$ CI $[-0.05,0.16]$ (5\% fat).

Does framing effect size vary across quantity pairs? We tested whether the framing effect would be larger for the $25 \%$ fat pair than the $5 \%$ fat one in a preregistered ANOVA with frame and quantity pair as factors. As predicted, this effect was greater in the $25 \%$ fat than the $5 \%$ fat condition, $F(1,436)=122.71, p<.001$, $\eta 2 \mathrm{P}$ $=.22$.

\section{Discussion}

Experiment 3 showed a similar pattern to Experiment 2. We observed a smaller framing effect for the $5 \%$ fat pair than the $25 \%$ fat one, with no significant evidence that quantifier format moderated this effect. In addition, the focus on a lean attribute partially mediated the relationship between frame and healthiness judgement for the $25 \%$ fat pair, suggesting that for 25 (a moderate) $\%$ fat and 75 (a high) $\%$ lean, the focus was on the cited attribute and hence contributed to the framing effect. However, the mediation was not observed for the $5 \%$ fat pair, likely because the quantifier frames in this pair had opposing foci: 95 (a very high) \% lean focused on the lean attribute, and 5 (a low) \% fat also directed focus to the lean attribute-away from the fat attribute. This focus on attribute or complement was not significantly 
different between verbal and numerical quantifiers, contrasting with previous findings that verbal probabilities possessed less ambiguous focusing properties than numerical ones (Teigen \& Brun, 1995, 2000).

\section{Framing Effect and the Moderating Role of Format: Data Synthesis Across \\ Experiments}

Across three experiments, we found inconsistent evidence regarding the role of format on the framing effect magnitude so to further evaluate the role of format as a moderator, we meta-analysed the moderated framing effect for the $5 \%$ fat and $25 \%$ fat pairs across the three experiments reported here. Meta-analytical methods provide more precision in the estimation and minimise the chance of obtaining null effects due to lack of statistical power (Cumming, 2013). We computed the internal meta-analysis using random effect models (a restricted maximum likelihood estimator) with the $\mathrm{R}$ package 'metafor' (Viechtbauer, 2010).

The overall framing effect was significant (see Figure 4), $b=0.75, p<.001$, $95 \%$ CI [0.42, 1.07], and format was not a significant moderator across studies for either quantity magnitude, $b=0.01, p=.934,95 \%$ CI $[-0.31,0.33]$ (5\% fat); $b=0.12$, $p=.643,95 \%$ CI $[-0.40,0.65](25 \%$ fat $)$. 


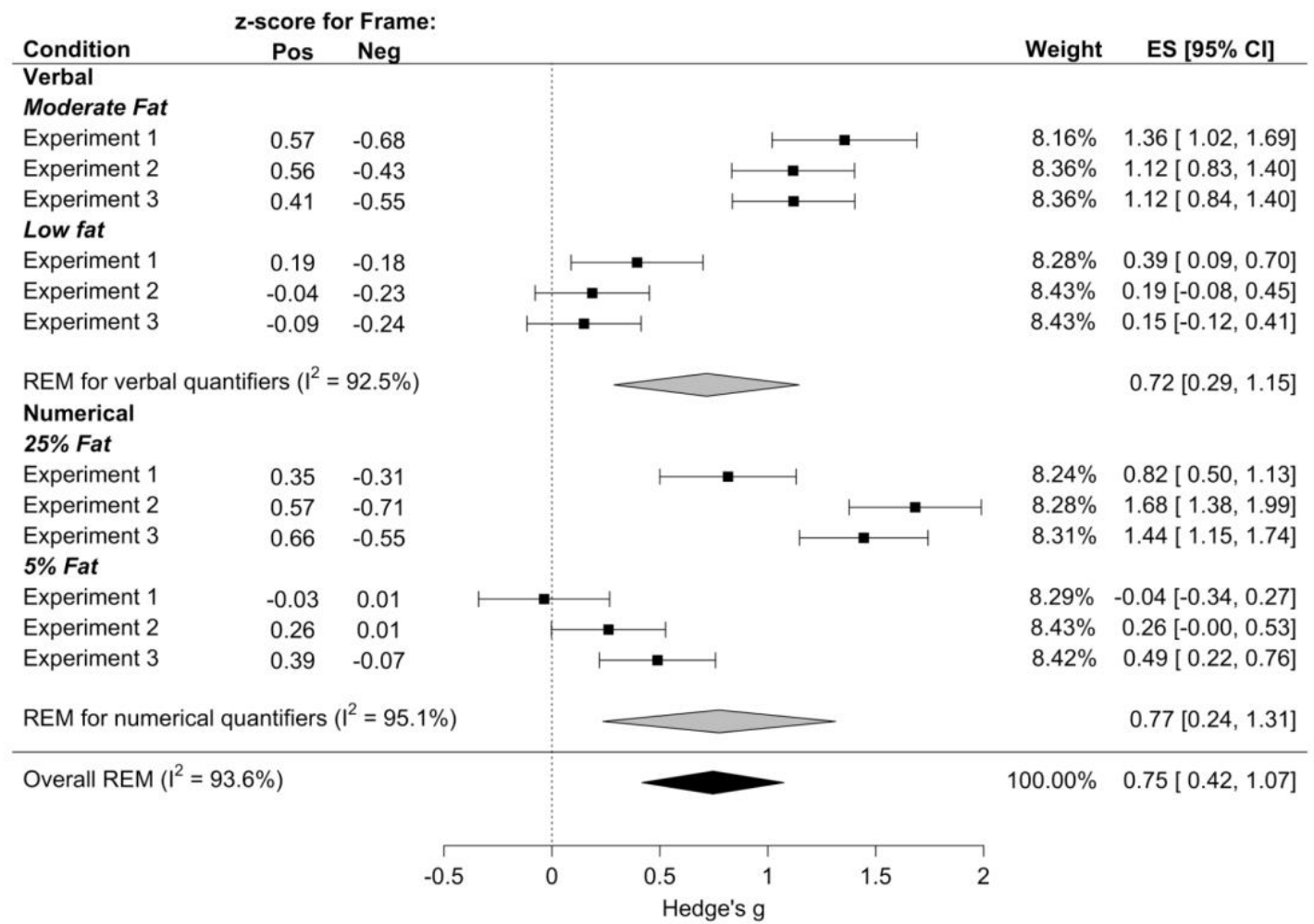

Figure 4. Forest plot of the framing effect sizes for verbal and numerical conditions across three experiments showing similar framing magnitude across formats.

Note. The grey diamonds show the random effects model for verbal and numerical quantifiers, and the black diamond shows the overall effect size across all formats and quantity pairs.

\section{General Discussion}

In three experiments, we investigated how the format of a quantifier moderates the attribute framing effect across different quantity pairs, and whether the amount of focus on the attribute in the frame could explain the effects. Across the three experiments, we replicated the traditional framing effect, showing that beef described in terms of its lean content was judged healthier than beef described with an equivalent fat content, but we did not find evidence that verbal quantifiers magnify the framing effect. 


\section{No effects of quantifier format: Implications for previous empirical findings}

Contrary to our predictions and previous empirical findings (Welkenhuysen et al., 2001), the effect of quantifier format was inconsistent, with only one quantity pair showing a magnified verbal framing effect in one experiment. Our meta-analysis across three well-powered experiments indicated that the hypothesised moderated framing effect was not present in the original attribute framing design we used (Levin, 1987), even when controlling for individual variations in how people translate between numerical and verbal quantifiers. Previous findings of a larger framing effect with verbal quantifiers (Welkenhuysen et al., 2001) may have reflected translation differences between a pre-test and experimental sample, or the specificity of the context in which it was tested. We used a known framing design in the context of fat and lean meat so that we could test our predictions systematically in a scenario with a replicable framing effect. In order to keep the sentences structures consistent across the numerical and verbal framing scenarios, we described a beef in terms of how much fat meat or lean meat it contained. This is slightly different from the standard construction of $25 \%$ fat or $75 \%$ lean beef — and indeed, some participants could have interpreted that the beef was comprised of fat and meat (as opposed to 'fat meat'). However, we still produced the classic framing effect throughout, which suggests that this amendment to the sentence construction was not critical to the framing effect.

Because our participants translated the numerical quantifiers into verbal quantifiers and performed the framing task within the same context, we predict that the same method (controlling for translation differences) would produce similar results with materials where the numerical complements might have different meanings for people. For example, $5 \%$ fat and $95 \%$ lean meat may be a low and very high amount, but 5\% fat and 95\% non-fat milk may be a moderate and high amount. 
Taking into consideration the difference in translations, though, one would just compare the numerical frames to the corresponding moderate fat and high non-fat frames. However, further investigations should still test whether a context like the $5 \%$ fat vs. $95 \%$ non-fat milk would produce the moderating effect of format in attribute framing.

\section{Implications about quantifier properties and framing effects}

We predicted that verbal quantifiers would magnify the framing effect compared to numerical ones based on two posited properties of verbal quantifiers. First, verbal quantifiers are believed to be processed more intuitively and lead to more judgement biases (Windschitl \& Wells, 1996). Second, verbal quantifiers should focus people's attention more strongly on the attribute compared to numerical quantifiers (Moxey, 2017; Teigen \& Brun, 1995, 2000). This would lead to greater pragmatic signals that this attribute is important to the judgement. Finding that the quantifier format did not moderate the framing effect could either mean lack of evidence that these properties differ between verbal and numerical quantifiers, or lack of evidence for the assumption that these properties produce the framing effect. To address this issue, we examine two findings of our data: the variations in framing effect size between different quantity pairs, and the mediation of the framing effect by a focus on the lean attribute (vs. the fat one).

Framing effect size varied across different quantity pairs. We replicated the classic framing effect, with similar effect sizes between verbal and numerical quantifiers. Instead, the variations in effect sizes came from differences in the quantity pairs: the $25 \%$ fat pair consistently produced the largest effect. This supports work that found effect size variations across frames with different quantity pairs (Janiszewski et al., 2003; Kim et al., 2014; Sanford et al., 2002), but contrasts with 
work that did not (Jin et al., 2017, Olsen, 2015). Assuming that the framing effect is an intuitive bias driven by an initial affect response to the positive or negative frame (Levin, 1987), one would expect a similar framing effect size irrespective of the exact quantity pair because the association created by 'fat' (or 'lean') is present in every pair. The differences in effect sizes between quantity pairs like $25 \%$ vs. $5 \%$ fat (75\% vs. 95\% lean) suggests people integrate it into their judgements. This could be because the quantifier automatically scales the affective reaction to the frame, but if this were the case, we would also expect to see scaling extend similarly across frames: moderate (or 25\%) fat might be more negative than low (or 5\%) fat, but very high (or 95\%) lean should also be more positive than high (or 75\%) lean. Even if the scaling is asymmetric for positive and negative frames (e.g., people are more averse to losses than they are receptive to gains; Tversky \& Kahneman, 1991), we should expect to observe this asymmetry in the quantity pairs in Experiment 1 . The negative-ness of $50 \%$ fat and $75 \%$ fat should loom larger than the positive-ness of $50 \%$ and $25 \%$ lean, which should have produced a larger framing effect than for the $25 \%$ fat (or $75 \%$ lean) pair. However, this was not the case in Experiment 1. It is also worth noting that when translating numerical to verbal quantifiers, both $50 \%$ and $75 \%$ fat were most commonly described as 'very high', but different verbal quantifiers were used for all four quantities of lean meat. Participants thus seemed to be more sensitive to gradation in the lean attribute than the fat one. Whether participants focused on the attribute or its complement might therefore better explain the framing effect.

\section{Focus on the lean attribute partially mediated the framing effect. Our} other experimental goal was to investigate participants' focus on the attribute in the frame as an explanation for the framing effect. Based on the sentence completion task, our participants found explanations focusing on fat to be more reasonable for 25 
(moderate) $\%$ fat frames, but not for 5 (low) \% fat ones. Our mediation analyses then found that a greater focus on the lean attribute (compared to the fat one) explained the framing effect for the $25 \%$ fat pair but not the $5 \%$ fat one. This difference in focus and its mediating effect was observed for both verbal and numerical quantifiers.

Differences between quantifier pairs: A question of ambiguity? One key feature of the $25 \%$ fat pair that distinguishes it from the others is that it is a more ambiguous complement pairing, that may be less immediately informative about its position on a scale of healthiness (e.g., people may be uncertain about what exactly is a healthy level of fat; Diekman \& Malcolm, 2009). Our framing task involved some quantitative judgement to assess the beef on a scale of healthiness. People may thus have focused primarily on the quantifier and only increased focus on the frame depending on how ambiguous the quantifier was. People tend to draw more from implicit information (i.e., pragmatic inferences; Sher \& McKenzie, 2006) when they need to distinguish ambiguous targets (Grodner \& Sedivy, 2011). For example, one may have a vague idea of the range of fat quantities that might be considered healthy, but be uncertain whether 25 (or a moderate) \% falls within that range (Janiszewski et al., 2003). One might then rely on the implicit focus in the quantifier and frame to infer that $25 \%$ is a larger than usual amount of fat, and thus not so healthy (Donovan \& Jalleh, 1999; Sher \& McKenzie, 2006). This process would be reversed in the lean condition, resulting in a conclusion that the 75 (a high) \% lean beef is healthier. In contrast, a $5 \%$ fat (95\% lean) beef is more apparently a healthy quantity, meaning the frame and focus is less informative to the judgement. Although we did not formally assess people's existing knowledge of the typical range of fat in meat, it is reasonable to assume that 5 (a low) \% fat is more clearly healthy than $25 \%$ fat. Previous work 
the quantity should be higher (e.g., that 5 (low) \% was a lower than expected amount of fat; Moxey, 2006; Moxey \& Filik, 2010). A salient question for future research is whether manipulating ambiguity and expectations about a quantifier could eliminate or magnify the framing effect. This would help to ascertain the conditions under which focusing properties best explain the framing effect.

No difference in focus on the attribute between formats. We predicted, but did not find, that the verbal quantifiers would put different levels of focus on the attribute described in the frame. Our hypothesis was based on previous research that found numerical quantifiers to place less attention on their attributes than verbal quantifiers (Moxey, 2017), and numerical probabilities such as 'a 30\% chance' to be more ambiguous in their focus to attribute or complement than their average translated verbal probabilities (e.g., 'some possibility', which focuses strongly on the occurrence of an event; Teigen \& Brun, 2000). We used verbal quantifiers constructed in a way that typically puts focus on an attribute rather than its complement (e.g., 'a low \%', which is similar to 'a few'; Moxey \& Sanford, 1986). However, we found that 'a low $\%$ of fat meat' and ' $5 \%$ of fat meat' both produced a focus on the complementary lean meat.

There are several possible reasons why our findings were different. First, 'a low $\%$ of' does not have a natural equivalent of 'low $\%$ of', so we could not compare these constructions of the verbal quantifier. Past work has indicated that the term 'low fat' produces a complement focus (Sanford \& Moxey, 2003). We cannot be certain that this construction would have been even more focused on the complementary lean meat, but we believe it unlikely given that over $90 \%$ of our participants selected causal sentence completions that were focused on the complement. Rather, the verbal quantifier 'low' seems to be inherently focused on the absence of its attribute- 
possibly because it generates an expectation of that the amount is less than expected (Moxey, 2006).

Second, our expectation that the numerical quantifiers would be more ambiguous in whether they focused on the attribute or its complement was based on previous work that compared verbal probabilities with their average numerical meanings (Teigen \& Brun, 1995, 2000). Our studies compared individually-translated verbal and numerical quantifiers. Taking into account how each individual interprets a verbal quantifier might better reflect how they view the focus of the corresponding numerical one. Furthermore, verbal probabilities differ from other quantifiers in two ways: they are uncertain, and they have an inherent 'frame' (e.g., 'unlikely' is more obviously negative than 'low'). This could mean that verbal probabilities have more scope to add information and also possess more information than other verbal quantifiers. Whether this would be true for other types of verbal quantifiers (e.g., 'uncommon' vs. 'common'; 'few' vs. 'a few') could be addressed in future research that compares the focus between different types of verbal quantifiers and their numerical counterparts.

\section{Conclusion}

The three experiments reported in this paper showed that contrary to previous empirical findings, the size of the attribute framing effect was not affected by quantifier format. We found evidence that in the case of both verbal and numerical quantifiers, when participants focused on the cited attribute in both frames, this focus contributed to the framing effect. In contrast, when participants focused on the complement in one frame but the attribute in the other, the framing effect was reduced. Our results also underlined the role of the quantity magnitude: different pairs of quantities produced different framing effect sizes. This shows that people do 
integrate the quantifier into their judgement about the overall item, and do so in a reasonable fashion for the context of the information.

\section{Acknowledgements}

Funding: This work was partially supported by a small grant from the Experimental Psychology Society. 


\section{References}

Berry, D. C., Knapp, P. R., \& Raynor, T. (2002). Is 15 per cent very common? Informing people about the risks of medication side effects. The International Journal of Pharmacy Practice, 10, 149-151. doi: 10.1111/j.20427174.2002.tb00602.x

Berry, D. C. (2006). Verbal labels can triple perceived risk in clinical trials. Therapeutic Innovation and Regulatory Science, 40, 249-258. doi: $10.1177 / 009286150604000302$

Budescu, D. V., Por, H.-H., \& Broomell, S. B. (2012). Effective communication of uncertainty in the IPCC reports. Climatic Change, 113, 181-200. doi: $10.1007 / \mathrm{s} 10584-011-0330-3$

Budescu, D. V., Por, H.-H., Broomell, S. B., \& Smithson, M. (2014). The interpretation of IPCC probabilistic statements around the world. Nature Climate Change, 4, 508-512. doi: 10.1038/NCLIMATE2194

Cumming, G. (2013). The new statistics: Why and how. Psychological Science, 25, 729. doi: $10.1177 / 0956797613504966$

Diekman, C., \& Malcolm, K. (2009). Consumer perception and insights on fats and fatty acids: Knowledge on the quality of diet fat. Annals of Nutrition \& Metabolism, 54, 25-32. doi: 10.1159/000220824

Donovan, R. J., \& Jalleh, G. (1999). Positively versus negatively framed product attributes: The influence of involvement. Psychology and Marketing, 16, 613630. doi: 10.1002/(SICI)1520-6793(199910)16:7<613::AID-

MAR4>3.0.CO;2-F 
Gal, D., \& Rucker, D. (2018). Loss aversion, intellectual inertia, and a call for a more contrarian science: A reply to Simonson \& Kivetz and Higgins \& Liberman. Journal of Consumer Psychology. doi: 10.1002/jcpy.1044

Grice, H. P. (1975). Logic and conversation. In P. Cole \& J. L. Morgan (Eds.), Syntax and semantics, vol 3: Speech Arts (Vol. 3, pp. 41-58). New York, NY: Academic Press.

Grodner, D., \& Sedivy, J. C. (2011). The effect of speaker-specific information on pragmatic inferences. In E. A. Gibson \& N. J. Pearlmutter (Eds.), The processing and acquisition of reference (pp. 239-271). Cambridge, MA: MIT Press.

Isaac, M. S., \& Poor, M. (2016). The sleeper framing effect: The influence of frame valence on immediate and retrospective judgments. Journal of Consumer Psychology, 26, 53-65. doi: 10.1016/j.jcps.2015.05.002

Janiszewski, C., Silk, T., \& Cooke, A. J. (2003). Different scales for different frames: The role of subjective scales and experience in explaining attribute-framing effects. Journal of Consumer Research, 30, 311-325. doi: 10.1086/378612

Jin, J., Zhang, W., \& Chen, M. (2017). How consumers are affected by product descriptions in online shopping: Event-related potentials evidence of the attribute framing effect. Neuroscience Research, 125, 21-28. doi: 10.1016/j.neures.2017.07.006

Kahneman, D. (2003). A perspective on judgment and choice: Mapping bounded rationality. American Psychologist, 58, 697-720. doi: 10.1037/0003066X.58.9.697 
Keysar, B., Hayakawa, S. L., \& An, S. G. (2012). The foreign-language effect:

Thinking in a foreign tongue reduces decision biases. Psychological Science. doi: $10.1177 / 0956797611432178$

Kim, J., Kim, J. E., \& Marshall, R. (2014). Search for the underlying mechanism of framing effects in multi-alternative multi-attribute decision situations. Journal of Business Research, 67, 378-385. doi: 10.1016/j.jbusres.2012.12.024

Kreiner, H., \& Gamliel, E. (2017). The role of attention in attribute framing. Journal of Behavioral Decision Making. doi: 10.1002/bdm.2067

Krishnamurthy, P., Carter, P., \& Blair, E. (2001). Attribute framing and goal framing effects in health decisions. Organisational Behavior \& Human Decision Processes, 85, 382-399. doi: 10.1006/obhd.2001.2962

Leong, L. M., McKenzie, C. R. M., Sher, S., \& Müller-Trede, J. (2017). The role of inference in attribute framing effects. Journal of Behavioral Decision Making, 10, 21-32. doi: 10.1002/bdm.2030

Levin, I. P. (1987). Associative effects of information framing. Bulletin of the Psychonomic Society, 25, 85-86. doi: 10.3758/BF03330291

Levin, I. P., \& Gaeth, G. J. (1988). How consumers are affected by the framing of attribute information before and after consuming the product. Journal of Consumer Research, 15, 374-378. doi: 10.1086/209174

Levin, I. P., Schneider, S. L., \& Gaeth, G. J. (1998). All frames are not created equal: A typology and critical analysis of framing effects. Organizational Behavior and Human Decision Processes, 76, 149-188. doi: 10.1006/obhd.1998.2804

Liu, D., Juanchich, M., Sirota, M., \& Orbell, S. (2019). People overestimate verbal quantities of nutrients on nutrition labels Food Quality and Preference. doi: 10.1016/j.foodqual.2019.103739 
Liu, D., Juanchich, M., Sirota, M., \& Orbell, S. (2020). The intuitive use of contextual information in decisions made with verbal and numerical quantities. Quarterly Journal of Experimental Psychology. doi: 10.1177/1747021820903439

Mandel, D. R. (2014). Do framing effects reveal irrational choice? Journal of Experimental Psychology: General, 143, 1185-1198. doi: 10.1037/a0034207

Maule, J., \& Villejoubert, G. (2007). What lies beneath: Reframing framing effects. Thinking \& Reasoning, 13, 25-44. doi: 10.1080/13546780600872585

McKenzie, C. R., \& Nelson, J. D. (2003). What a speaker's choice of frame reveals: reference points, frame selection, and framing effects. Psychonomic Bulletin \& Review, 10, 596-602. doi: 10.3758/BF03196520

Moxey, L. M. (2006). Effects of what is expected on the focussing properties of quantifiers: A test of the presupposition-denial account. Journal of Memory and Language, 55, 422-439. doi: 10.1016/j.jml.2006.05.006

Moxey, L. M. (2017). Processing quantified noun phrases with numbers versus verbal quantifiers. Discourse Processes, 55, 136-145. doi: 10.1080/0163853X.2017.1330042

Moxey, L. M., \& Filik, R. (2010). The effects of character desire on focus patterns and pronominal reference following quantified statements. Discourse Processes, 47, 588-616. doi: 10.1080/01638530903572976

Moxey, L. M., \& Sanford, A. J. (1986). Quantifiers and focus. Journal of Semantics, 5, 189-206.

Naughton, P., McCarthy, S. N., \& McCarthy, M. B. (2015). The creation of a healthy eating motivation score and its association with food choice and physical activity in a cross sectional sample of Irish adults. International Journal of 
Behavioral Nutrition and Physical Activity, 12, 1-10. doi: 10.1186/s12966015-0234-0

Olsen, A. L. (2015). Citizen (dis)satisfaction: An experimental equivalence framing study. Public Administration Review, 75, 469-478. doi: 10.1111/puar.12337

Renkewitz, F., Fuchs, H. M., \& Fiedler, S. (2011). Is there evidence of publication biases in JDM research? Judgment and Decision Making, 6, 870-881.

Reyna, V. F., \& Brainerd, C. J. (1991). Fuzzy - trace theory and framing effects in choice: Gist extraction, truncation, and conversion. Journal of Behavioral Decision Making, 4, 249-262. doi: 10.1002/bdm.3960040403

Saad, G., \& Gill, T. (2014). The framing effect when evaluating prospective mates: An adaptationist perspective Evolution and Human Behavior, 35, 184-192. doi: 10.1016/j.evolhumbehav.2014.01.002

Sanford, A. J., Dawydiak, E. J., \& Moxey, L. M. (2007). A unified account of quantifer perspective effects in discourse. Discourse Processes, 44, 1-32. doi: $10.1080 / 01638530701285556$

Sanford, A. J., Fay, N., Stewart, A., \& Moxey, L. M. (2002). Perspective in statements of quantity, with implications for consumer psychology. Psychological Science, 13, 130-134. doi: 10.1111/1467-9280.00424

Sanford, A. J., \& Moxey, L. M. (2003). New perspectives on the expression of quantity. Current Directions in Psychological Science, 12, 240-243. doi: 10.1046/j.0963-7214.2003.01270.x

Seta, J. J., McCormick, M., Gallagher, P., McElroy, T., \& Seta, C. E. (2010). Voice frequency impacts hemispheric processing of attribute frames Journal of Experimental Social Psychology, 46, 1089-1092. doi:

10.1016/j.jesp.2010.06.009 
Sher, S., \& McKenzie, C. R. M. (2006). Information leakage from logically equivalent frames. Cognition, 101, 467-494. doi: 10.1016/j.cognition.2005.11.001

Sher, S., \& McKenzie, C. R. M. (2008). Framing effects and rationality. In N. Chater \& M. Oaksford (Eds.), The probabilistic mind: Prospects for Bayesian cognitive science (pp. 79-96). Oxford, UK: Oxford University Press.

Sher, S., \& McKenzie, C. R. M. (2014). Options as information: Rational reversals of evaluation and preference. Journal of Experimental Psychology: General, 143, 1127-1143. doi: 10.1037/a0035128

Steptoe, A., Pollard, T. M., \& Wardle, J. (1995). Development of a measure of the motives underlying the selection of food: The food choice questionnaire. Appetite, 25, 267-284. doi: 10.1006/appe.1995.0061

Teigen, K. H., \& Brun, W. (1995). Yes, but it is uncertain: Direction and communicative intention of verbal probabilistic terms. Acta Psychologica, 88, 233-258. doi: 10.1016/0001-6918(93)E0071-9

Teigen, K. H., \& Brun, W. (1999). The directionality of verbal probability expressions: Effects on decisions, predictions, and probabilistic reasoning. Organizational Behavior and Human Decision Processes, 80, 155-190. doi: 10.1006/obhd.1999.2857

Teigen, K. H., \& Brun, W. (2000). Ambiguous probabilities: When does $p=0.3$ reflect a possibility, and when does it express a doubt? Journal of Behavioral Decision Making, 13, 345-362. doi: 10.1002/10990771(200007/09)13:3<345::AID-BDM358>3.0.CO;2-U

Teigen, K. H., \& Brun, W. (2003). Verbal probabilities: A question of frame? Journal of Behavioral Decision Making, 16, 53-72. doi: 10.1002/bdm.432 
Teigen, K. H., Juanchich, M., \& Filkukova, P. (2014). Verbal probabilities: An alternative approach. Quarterly Journal of Experimental Psychology, 67, 124146. doi: $10.1080 / 17470218.2013 .793731$

Thomas, A. K., \& Millar, P. R. (2012). Reducing the framing effect in older and younger adults by encouraging analytic processing. Journal of Gerontology Series B Psychological Sciences and Social Sciences, 67, 139-149. doi: 10.1093/geronb/gbr076

Tingley, D., Yamamoto, T., Hirose, K., Keele, L., \& Imai, K. (2014). mediation: R Package for Causal Mediation Analysis. Journal of Statistical Software, 59. doi: $10.18637 /$ jss.v059.i05

Tversky, A., \& Kahneman, D. (1981). The framing of decisions and the psychology of choice. Science, 211, 453-458. doi: 10.1126/science.7455683

Tversky, A., \& Kahneman, D. (1991). Loss aversion in riskless choice: A referencedependent model. The Quarterly Journal of Economics, 106, 1039-1061.

van Buiten, M., \& Keren, G. (2009). Speakers' choice of frame in binary choice: Effects of recommendation mode and option attractiveness. Judgment and Decision Making, 4, 51-63.

Viechtbauer, W. (2010). Conducting meta-analyses in R with the metafor package. Journal of Statistical Software, 36, 1-48. doi: 10.18637/jss.v036.i03

Voutilainen, A., Pitkäaho, T., Kvist, T., \& Vehviläinen-Julkunen, K. (2016). How to ask about patient satisfaction? The visual analogue scale is less vulnerable to confounding factors and ceiling effect than a symmetric Likert scale. Journal of Advanced Nursing 72, 946-957. doi: 10.1111/jan.12875

Welkenhuysen, M., Evers-Kiebooms, G., \& d'Y dewalle, G. (2001). The language of uncertainty in genetic risk communication: Framing and verbal versus 
numerical information. Patient Education and Counseling, 43, 179-187. doi: $10.1016 / \mathrm{S} 0738-3991(00) 00161-0$

Windschitl, P. D., \& Wells, G. L. (1996). Measuring psychological uncertainty: Verbal versus numeric methods. Journal of Experimental Psychology: Applied, 2, 343-364. doi: 10.1037//1076-898X.2.4.343 


\section{Appendix}

Table A.

List of verbal quantifiers for translation task.

\begin{tabular}{cc}
\hline Verbal quantifier & Translation range \\
\hline Insignificant & Very low \\
Very low & Low \\
\hline Low & \\
Small & Medium \\
\hline Fair & \\
Medium & High \\
Moderate & \\
\hline Large & \\
High & Very high \\
\hline Very large & \\
Very high & \\
\hline
\end{tabular}

